\title{
On contingent equations satisfying the Carathéodory type conditions
}

\author{
By \\ Norio KIKUCH*
}

\section{Introduction}

In [1] we have proved a bang-bang type property for the nonlinear contingent equation which is continuous in two variables. We shall prove the same property for the Carathéodory type contingent equation. For this proof we use a proposition which roughly says that the Carathéodory type function is approximately continuous in two variables.

First we prove this property for the set-valued function. While preparing this manuscript, the author noticed that a similar theorem had been proved in [3] for the vector-valued function. Also in [2] this property has been used in order to prove that the Carathéodory type differential equation has absolutely continuous solutions whose derivatives exist at almost every point common to all these solutions. We extend this theorem to the Carathéodory type contingent equation.

The author wishes here to express his thanks to Professor Masuo Hukuhara for his suggestion of the before mentioned problem and his warm encouragement.

\section{Notations and definitions}

We denote by $R^{m}$ an $m$-dimensional Euclidean space.

By

$$
\operatorname{Comp}\left(R^{m}\right), \quad\left(\operatorname{resp} . \operatorname{Conv}\left(R^{m}\right)\right)
$$

Received October 27, 1967.

Communicated by M. Hukuhara.

* Department of Mathematics, Kôbe University. 
we denote the collection of all nonempty compact (resp. compact and convex) subsets of $R^{m}$.

By

$$
\operatorname{dist}(x, y) \text { and } \operatorname{dist}(x, A)=\inf \{\operatorname{dist}(x, y) ; y \in A\}
$$

we denote the distance of a point $x$ from a point $y \in R^{m}$ and a set $A \subset R^{m}$, respectively.

$$
\operatorname{Dist}(A, B)=\inf \{s>0 ; A \subset V(B, s), B \subset V(A, s)\}
$$

is the Hausdorff's distance of two compact sets $A$ and $B$, where $V(A, s)$ denotes the closed neighborhood of a set $A$ of radius $s$, i.e.

$$
V(A, s)=\left\{x \in R^{m} ; \operatorname{dist}(x, A) \leqq s\right\} .
$$

For a set $A$ in $R^{m}$ we put

$$
|A|=\operatorname{Dist}(O, A) \text {, }
$$

where $O$ is the origin of $R^{m}$ and we denote by bdry $A$ the boundary of $A$.

Let $T$ be a topological and measurable space. A map $N(t)$ of $T$ into $\operatorname{Comp}\left(R^{m}\right)$ will be called an orientor field.

The continuity of the orientor field $N(t)$ in the sense of Hausdorff is defined in a usual way.

$N(t)$ is said to be measurable on $T$ if the set $\{t \in T ; N(t) \subset C\}$ is measurable for every $C \in \operatorname{Comp}\left(R^{m}\right)$.

For a measurable set $E$ we denote by meas $(E)$ the measure of $E$. The abbreviation a.e. $E$ means almost everywhere in $E$.

Let $I$ be a compact interval $\left[t_{0}, t_{0}+a\right]$ and $x(t) \in R^{m}$ be a function defined on $I$.

Let $g$ be a vector in $R^{m}$ for which there exists a sequence $\left\{t_{i}\right\} \rightarrow t, t_{i} \neq t, t_{i} \in I$ such that

$$
\left(x\left(t_{i}\right)-x(t)\right) /\left(t_{i}-t\right) \rightarrow g \quad \text { as } i \rightarrow \infty .
$$

The set of all such $g$ will be called the contingent derivative of $x(t)$ at $t$ and we denote it by $D^{*} x(t)$.

For the function $F(t, x) \in \operatorname{Conv}\left(R^{m}\right)$ defined on $I \times R^{m}$ we assume the following 
Hypothesis $\mathbf{H}(\mathbf{F}) . F(t, x)$ is measurable in $t$ for each fixed $x \in R^{m}$ and continuous in $x$ for each fixed $t \in I$. There exists an integrable function $k(t) \geqq 0$ such that

$$
|F(t, x)| \leqq k(t) \quad \text { a.e. } I .
$$

An absolutely continuous function $x(t)$ defined on $I$ will be called a trajectory of $F(t, x)$ if the following relation holds

$$
d x(t) / d t \in F(t, x(t)) \quad \text { a.e. } I \text {. }
$$

For a set $A$ in $R^{m}$ we denote by

$$
Z(A, F)
$$

the union of the graphs of trajectories $x(t)$ (on $I$ ) of $F(t, x)$ which satisfy the initial condition $x\left(t_{0}\right) \in A$.

\section{Propositions}

Proposition 1. Let $\left\{F_{n}(t)\right\}(n=1,2, \cdots)$ be a sequence of compactset valued functions defined and measurable on a measurable set $E$, and suppose that $\lim _{n \rightarrow \infty} F_{n}(t)=F(t)$ and meas $(E)<\infty$. Then for every positive number $\varepsilon$ we can choose a compact set $E_{\epsilon}$ such that $\left\{F_{n}(t)\right\}$ converges uniformly to $F(t)$ on $E_{\varepsilon}$, and meas $\left(E-E_{\varepsilon}\right)<\varepsilon$.

Proof. For every positive number $\lambda$ we put

$$
E_{n}(\lambda)=\bigcap_{k=1}^{\infty}\left\{t \in E ; \operatorname{Dist}\left(F_{n+k}(t), F(t)\right)<\lambda\right\} .
$$

$E=\bigcup_{n=1}^{\infty} E_{n}(\lambda)$ holds from the hypothesis. Since $E_{n}(\lambda) \subset E_{n+1}(\lambda)(n=$ $1,2, \cdots)$ hold,

$$
\lim _{n \rightarrow \infty} \operatorname{meas}\left(E_{n}(\lambda)\right)=\operatorname{meas}\left(\bigcup_{n=1}^{\infty} E_{n}(\lambda)\right)=\operatorname{meas}(E) .
$$

Hence for every positive number $\eta$ we can choose an integer $N$ such that

$$
\operatorname{meas}\left(E-E_{N}(\lambda)\right)<\eta \text {. }
$$

The corresponding $N$ and $E_{N}(\lambda)$ to $\lambda=1 / p, \eta=2^{-p} \varepsilon$ are denoted respectively by $N_{p}$ and $H_{p}$. 
From the definition of $H_{p}$ and $N_{p}$ we have

$$
\operatorname{Dist}\left(F_{n}(t), F(t)\right)<1 / p, \operatorname{meas}\left(E-H_{p}\right)<2^{-\star *} \varepsilon
$$

for every $t \in H_{p}$ and every $n \geqq N_{p}$. We put

$$
H=\bigcap_{p=1}^{\infty} H_{p},
$$

and then we have

$$
E-H=\bigcup_{p=1}^{\infty}\left(E-H_{p}\right), \operatorname{meas}(E-H) \leqq \sum_{p=1}^{\infty} \operatorname{meas}\left(E-H_{p}\right)<\varepsilon .
$$

Let $\delta$ be any positive number and we can chocse an integer $p$ such that

$$
1 / p \leqq \delta<1 /(p-1) .
$$

If $t \in H, t \in H_{p}$ for $p$ and

$$
\operatorname{Dist}\left(F_{n}(t), F(t)\right)<1 / p \leqq \delta
$$

holds for every $n \geqq N_{p}$. $N_{p}$ depends only on $\delta$ but not on $x$ in $H$ and hence $\left\{F_{n}(t)\right\}$ converges uniformly on $H$. Further we can choose in $H$ a compact set $E_{\varepsilon}$ such that meas $\left(H-E_{\varepsilon}\right)$ is as small as desired. This completes the proof of Proposition 1.

Proposition 2. Let $k(t)$ be a nonnegative function which is defined and integrable on an interval $I$, and let $E$ be a measurable subset of $I$. Then for almost every $\tau$ in the complement of $E$ with respect to $I$

$$
\lim _{h \rightarrow 0} \frac{1}{h} \int_{E_{h}(\tau)}^{k}(t) d t=0
$$

holds, where $E_{h}(\tau)=E \cap[\tau, \tau+h]$.

Proof. We define $\vec{k}(t)$ equal to $k(t)$ in $E$ and to zero in the complement of $E$. Then for almost every $\tau$ in $I$

$$
\lim _{h \rightarrow 0} \frac{1}{h} \int_{\tau}^{\tau+h} \bar{k}(t) d t=\bar{k}(\tau)
$$

holds, and hence for almost every $\tau$ in the complement of $E$

$$
\lim _{h \rightarrow 0} \frac{1}{h} \int_{E_{h}(\tau)} k(t) d t=0
$$

holds. 
Proposition 3. Let $K$ be a compact rectangle in $R^{m}$ and $E$ be a measurable set in $R^{1}$. If $F(t, x) \in \operatorname{Comp}\left(R^{m}\right)$ is defined on $E \times K$ and measurable in $t$ for each fixed $x \in K$ and continuous in $x$ for each fixed $t \in E$, then for every positive number $\varepsilon$ there is a compact subset $E_{\varepsilon}$ of $E$ such that meas $\left(E-E_{\varepsilon}\right)<\varepsilon$ and such that the function $F(t, x)$, when considered as defined on $E_{\varepsilon} \times K$ only, is uniformly continuous in $(t, x)$.

Proof. Let $K$ be the rectangle consisting of points $\left(x_{1}, x_{2}, \cdots, x_{m}\right)$ in $R^{m}$ such that

$$
a_{i} \leqq x_{i} \leqq b_{i}, i=1,2, \cdots, m \text {. }
$$

For each $n(n=1,2, \cdots)$ we now divide each interval $\left[a_{i}, b_{i}\right]$, $1 \leqq i \leqq m$, by points

$$
a_{i}=x_{i}^{(0)}<\cdots<x_{i}^{\left(2^{n}\right)}=b_{i},
$$

where $x_{i}^{(l)}=a_{i}+l\left(b_{i}-a_{i}\right) / 2^{n}$, and we get $2^{\text {mn }}$ congruent compact subrectangles $K_{n, k}\left(k=1,2, \cdots, 2^{m n}\right)$ whose barycenter we will denote by $x_{n, k}$. We put

$$
\rho_{n}=\min \left\{\left(b_{i}-a_{i}\right) / 2 n ; 1 \leqq i \leqq m\right\} .
$$

Let $F_{n, k}(t)$ be the oscillation of $F(t, x)$ in $K_{n, k}$ with respect to $x$, i. e.

$$
F_{n, k}(t)=\sup \left\{\operatorname{Dist}(F(t, x), F(t, y)) ; x, y \in K_{n, k}\right\},
$$

and we put

$$
\bar{F}_{n}(t)=\max \left\{F_{n, k}(t) ; k, 1 \leqq k \leqq 2^{m n}\right\} .
$$

Then from the continuity of $F(t, x)$ in $x, \bar{F}_{n}(t)$ can be verified to be measurable on $E$ and also we conclude that

$$
\lim _{n \rightarrow \infty} \bar{F}_{n}(t)=0
$$

Let $\varepsilon$ be an arbitrary positive number. We take a sequence of positive numbers $\left\{\varepsilon_{i}\right\}(i=1,2, \cdots)$ such that

$$
\varepsilon / 2=\sum_{i=1}^{\infty} \varepsilon_{i} .
$$

Since $F\left(t, x_{1, k}\right)\left(k=1,2, \cdots, 2^{m}\right)$ are measurable on $E$, we can select a 
compact set $E_{1}$ such that $F\left(t, x_{1, k}\right)$, restricted to $E_{1}$, are continuous in $t$, and meas $\left(E-E_{1}\right)<\varepsilon_{1}$. We define inductively $\left\{E_{n}\right\}(n=1,2, \cdots)$ as follows. Suppose that we have defined $E_{n}$ such that $F\left(t, x_{p, k}\right) \quad\left(k=1,2, \cdots, 2^{p m}\right.$, $p=1,2, \cdots, n)$ are continuous on $E_{n !}$, and meas $\left(E-E_{n}\right)<\sum_{i=1}^{n} \varepsilon_{i}$, then, since $F\left(t, x_{n+1, k}\right)\left(k=1,2, \cdots, 2^{(n+1) m}\right)$ are measurable on $E_{n}$, we can find a compact subset $E_{n+1}$ of $E_{n}$ such that $F\left(t, x_{n+1, k}\right)\left(k=1,2, \cdots, 2^{(n+1) m}\right)$ are continuous on $E_{n+1}$, and meas $\left(E_{n}-E_{n+1}\right)<\varepsilon_{n+1}$. We put

$$
E_{\varepsilon}^{\prime}=\bigcap_{n=1}^{\infty} E_{n} \text {. }
$$

$E_{\varepsilon}^{\prime}$ is a compact set and we have

$$
\operatorname{meas}\left(E-E_{\varepsilon}{ }^{\prime}\right) \leqq \sum_{n=1}^{\infty} \text { meas }\left(E-E_{n}\right) \leqq \sum_{n=1}^{\infty} \varepsilon_{n}=\varepsilon / 2 .
$$

$F\left(t, x_{p, k}\right)\left(k=1,2, \cdots, 2^{p m}, p=1,2, \cdots\right)$ are all continuous on $E_{\varepsilon}^{\prime}$.

Further by Egoroff's Theorem we can find a compact subset $E_{\varepsilon}$ of $E_{\varepsilon}^{\prime}$ such that $\left\{\bar{F}_{n}(t)\right\}$ converges uniformly to zero on $E_{\varepsilon}$, and meas $\left(E_{\varepsilon}^{\prime}-E_{\varepsilon}\right)<\varepsilon / 2$.

Let $\eta$ be an arbitrary positive number. From the uniform convergence of $\left\{\bar{F}_{n}(t)\right\}$ on $E_{\varepsilon}$ we can find a positive integer $N$ such that $3 \bar{F}_{N}(t)<\eta / 2^{m}$ for every $t$ on $E_{\varepsilon}$. Take any $\left(t_{0}, x_{0}\right) \in E_{\varepsilon} \times K$, and then we can find a set

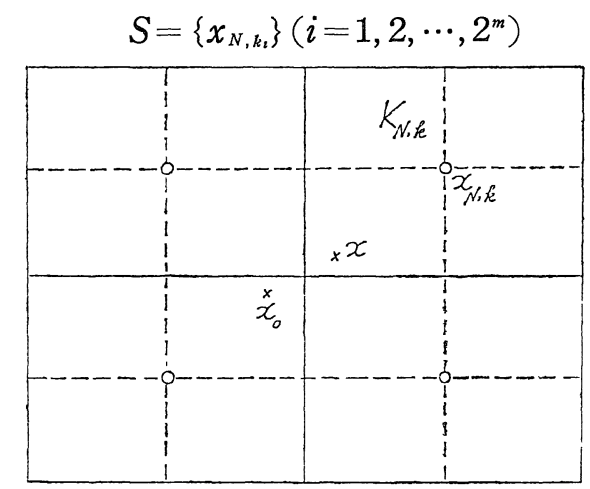

such that

$$
x \in K_{N, k i}
$$

for every $x \in K, \operatorname{dist}\left(x, x_{0}\right)<\rho_{N+1}$. Hence the relation

$$
\operatorname{Dist}(F(t, x), F(t, S))<2^{m} \bar{F}_{N}(t)<\eta / 3
$$


holds for every $t \in E_{\varepsilon}$ and $x \in K$, $\operatorname{dist}\left(x, x_{0}\right)<\rho_{N+1}$. Further we can find a positive number $\delta$ such that

$$
\operatorname{Dist}\left(F(t, S), F\left(t_{0}, S\right)\right)<\eta / 3
$$

holds every $t \in E_{\varepsilon},\left|t-t_{0}\right|<\delta$. Consequently if $(t, x) \in E_{\varepsilon} \times K$ satisfies

$$
\operatorname{dist}\left(x, x_{0}\right)<\rho_{N+1},\left|t-t_{0}\right|<\delta,
$$

the following relations hold

$$
\begin{aligned}
& \operatorname{Dist}\left(F(t, x), F\left(t_{0}, x_{0}\right)\right) \\
\leqq & \operatorname{Dist}(F(t, x), F(t, S))+\operatorname{Dist}\left(F(t, S), F\left(t_{0}, S\right)\right) \\
+ & \operatorname{Dist}\left(F\left(t_{0}, S\right), F\left(t_{0}, x_{0}\right)\right) \\
\leqq & 2^{m} \bar{F}_{N}\left(t_{0}\right)+\operatorname{Dist}\left(F(t, S), F\left(t_{0}, S\right)\right)+2^{m} \bar{F}_{N}\left(t_{0}\right)<\eta .
\end{aligned}
$$

Since $E_{\varepsilon} \times K$ is a compact set, $F(t, x)$ is uniformly continuous on $E_{\varepsilon} \times K$. From the construction of $E_{\varepsilon}$ the relation meas $\left(E-E_{\varepsilon}\right)<\varepsilon$ holds.

\section{Theorems}

Theorem 1. Let $F(t, x)$ satisfy the hypothesis $H(F)$. Then for every trajectory $x(t)$ of $F(t, x)$ which lies on bdry $Z(A, F)$ for every $t$ in $I, d x(t) / d t$ is on bdry $F(t, x(t))$ for almost every $t$ in $I$.

Proof. We can find a compact rectangle $K$ such that $Z(A, F)$ is contained in $I \times K$. Let $L$ be an integral of $k(t)$ on $I$.

Let $D_{n}$ be the set of the density points of $E_{1 / n}$, where $E_{1 / n}$ is as in Proposition 3. $D=\bigcup_{n=1}^{\infty} D_{n}$ is a subset of $I$ whose measure is equal to that of $I$. If $t$ belongs to $D$, there exists an integer $n$ such that $t \in D_{n}$. Then $t$ is a density point of $E_{1 / n}$ and $F(t, x)$, considered on $E_{1 / n} \times K$, is continuous in $(t, x)$.

Let $t_{1}$ be a point of $D$ such that $d \bar{x}\left(t_{1}\right) / d t$ belongs to the interior of $F\left(t_{1}, \bar{x}\left(t_{1}\right)\right)$ for some trajectory $\bar{x}(t)$ of $F(t, x)$ which lies on bdry $Z(A, F)$ for every $t$ in $I$, and so $V\left(d \bar{x}\left(t_{1}\right) / d t, 4 \varepsilon\right)$ is contained in $F\left(t_{1}, \bar{x}\left(t_{1}\right)\right)$ for some positive number $\varepsilon$. 
Let $\left[\tau, t_{1}\right]$ be a union $P \cup Q$ of two measurable sets $P, Q$. If the ratio

$$
\operatorname{meas}(Q) / \operatorname{meas}(P)
$$

is sufficiently small with $t_{1}-\tau$, there exists a positive number $\delta_{1}$ such that the following relation

$$
\int_{P} V\left(d \bar{x}\left(t_{1}\right) / d t, 3 \varepsilon\right) d t+\int_{Q} f(t) d t \supset \supset \int_{\tau}^{t_{1}} V\left(d \bar{x}\left(t_{1}\right) / d t, 2 \varepsilon\right) d t
$$

holds for $\tau, t_{1}-\delta_{1} \leqq \tau<t_{1}$, where $f(t)$ is a measurable function which lies in $F(t, x(t))$ for any trajectory $x(t)$ of $F(t, x)$.

On the other hand, since $\bar{x}(t)$ is differentiable at $t_{1}$, there exists a positive number $\delta_{2}$ such that the relation

$$
\left(\bar{x}(\tau)-\bar{x}\left(t_{1}\right)\right) /\left(\tau-t_{1}\right) \in V\left(d \bar{x}\left(t_{1}\right) / d t, \varepsilon\right)
$$

holds for every $\tau, t_{1}-\delta_{2} \leqq \tau<t_{1}$.

We take $\tau$ such that

$$
t_{1}-\delta \leqq \tau<t_{1}
$$

where $\delta=\min \left(\delta_{1}, \delta_{2}\right)$.

As stated above, in $\left[\tau, t_{1}\right]$ there exists a compact set $E_{1 / n} \cap\left[\tau, t_{1}\right]$, which we will denote by $J$, such that $t_{1}$ is a density point of $J$ and $F(t, x)$ is continuous in $(t, x)$, when it is considered on $J \times K$ only. By taking $\tau$ sufficiently near $t_{1}$, we can assume that $V\left(d \bar{x}\left(t_{1}\right) / d t, 3 \varepsilon\right)$ is contained in $F(t, x(t))$ for every $t \in J$ and for every function $x(t)$ which has the Lipschitz constant $L$ and coincides with $\bar{x}(t)$ at $\tau$ 。 $\left[\tau, t_{1}\right]$ can be expressed as a union of $J$ and $\bigcup_{i=1}^{\infty} I_{i}$, where $\left\{I_{i}\right\}(i=1,2, \cdots)$ is a sequence of compact intervals.

The union $\bigcup_{i=1}^{n} I_{i}$ is composed of a finite number of disjoint compact intervals which we will denote by $\left[\tau_{1}, \sigma_{1}\right],\left[\tau_{2}, \sigma_{2}\right], \cdots$ from left to right. The complement of $\bigcup_{i=1}^{n} I_{i}$ with respect $\left[\tau, t_{1}\right]$ is composed of a finite number of disjoint intervals which we will denote by $J_{1}=\left(\sigma_{1}, \tau_{2}\right), J_{2}=$ $\left(\sigma_{2}, \tau_{3}\right), \cdots$ or by $J=\left[\tau, \tau_{1}\right), J_{2}=\left(\sigma_{1}, \tau_{2}\right), \cdots$ according as $\tau_{1}=\tau$ or $\tau_{1} \neq \tau$.

Let $k$ be in $V\left(d \bar{x}\left(t_{1}\right) / d t, 3 \varepsilon\right)$. We define a curve on $\left[\tau, t_{1}\right]$ as follows. If $\tau \neq \tau_{1}$, we define on $\left[\tau, \tau_{1}\right]$

$$
x_{n}^{(k)}(t)=\bar{x}(\tau)+k(t-\tau) \text {. }
$$


On $\left[\tau_{1}, \sigma_{1}\right]$ we define $x_{n}^{(k)}(t)$ as a solution of the contingent equation through $\bar{x}(\tau)+k\left(\tau_{1}-\tau\right)$. On $\left[\sigma_{1}, \tau_{2}\right]$ we define

$$
x_{n}^{(k)}(t)=x_{n}^{(k)}\left(\sigma_{1}\right)+k\left(t-\sigma_{1}\right) \text {. }
$$

Similarly, if $\tau=\tau_{1}$, we define $x_{n}^{(k)}(t)$ as follows. On $\left[\tau, \sigma_{1}\right]$ we define $x_{n}^{(k)}(t)$ as a solution of the contingent equation through $\bar{x}(\tau)$. On $\left[\sigma_{1}, \tau_{2}\right]$ we define

$$
x_{n}^{(k)}(t)=x_{i n}^{(k)}\left(\sigma_{1}\right)+k\left(t-\sigma_{1}\right) .
$$

In this way we define a curve $x_{n}^{(k)}(t)$ on the whole interval $\left[\tau, t_{1}\right]$.

When $k$ goes round all over the set $V\left(d \bar{x}\left(t_{1}\right) / d t, 3 \varepsilon\right)$, we get a family of curves. This family can be verified to be a normal family and to have the same Lipschitz constant $L$.

From the construction of $\delta\left(\delta \leqq \delta_{1}\right)$, the set

$$
G=\left\{\bar{x}(\tau)+k\left(t_{1}-\tau\right) ; k \in V\left(d \bar{x}\left(t_{1}\right) / d t, 2 \varepsilon\right)\right\}
$$

is contained in the set

$$
G_{n}=\left\{x_{n}^{(k)}\left(t_{1}\right) ; k \in V\left(d \bar{x}\left(t_{1}\right) / d t, 3 \varepsilon\right)\right\}
$$

for sufficiently large $n$. Hence, for every $x \in G$ there exists a curve $x_{n}^{(k)}(t)$, which we denote by $x_{n}(t)$, such that $x_{n}\left(t_{1}\right)=x$. We can select a subsequence of $\left\{x_{n}(t)\right\}$ which converges uniformly to a curve $x^{*}(t)$ with the Lipschitz constant $L$. Since the relation

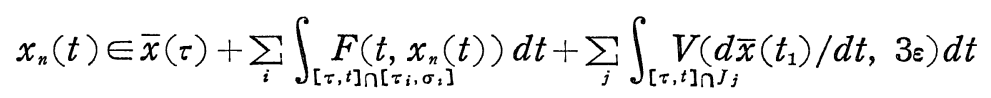

holds for every $n$, we have

$$
\begin{aligned}
x^{*}(t) & \in \bar{x}(\tau)+\int_{[\tau, t]-J} F\left(t, x^{*}(t)\right) d t+\int_{[\tau, t] \cap J} V\left(d \bar{x}\left(t_{1}\right) / d t, 3 \varepsilon\right) d t \\
& \subset \bar{x}(\tau)+\int_{\tau}^{t} F\left(t, x^{*}(t)\right) d t .
\end{aligned}
$$

This relation implies

$$
x^{*}(\tau)=\bar{x}(\tau)
$$

and

$$
d x^{*}(t) / d t \in F\left(t, x^{*}(t)\right) \quad \text { a.e. }\left[\tau, t_{1}\right]
$$


because $x^{*}(t)$ is absolutely continuous. $x^{*}(t)$ is a trajectory which connects $A$ and $x$. Hence $G$ is contained in $Z(A, F)$, and hence $x\left(t_{1}\right)$ belongs to the interior of $Z(A, F)$, which is a contradiction.

Theorem 2. Let $F(t, x)$ satisfy the hypothesis $H(F)$. Then every trajectory of $F(t, x)$ satisfies the contingent equation almost everywhere in I except for a common set of measure zero.

Proof. For any positive number $\varepsilon$ we let $E$ be a set $E_{\varepsilon}$ as in Proposition 3. Let $H$ be the complement of $E$ with respect to $I$ and $E^{\prime}$ be the set of density points of $E$ and $E^{\prime \prime}\left(\subset E^{\prime}\right)$ be the set of $\tau$ for which there holds

$$
\lim _{h \rightarrow 0} \frac{1}{h} \int_{H_{h}(\tau)} k(t) d t=0,
$$

as in Proposition 2. We get

$$
\text { meas }(E)=\text { meas }\left(E^{\prime}\right)=\text { meas }\left(E^{\prime \prime}\right)>\text { meas }(I)-\varepsilon \text {. }
$$

Let $\tau$ belong to $E^{\prime \prime}$ and $x(t)$ be any trajectory of the contingent equation. For a sufficiently small $h$ we get

$$
\frac{x(\tau+h)-x(\tau)}{h} \in \frac{1}{h} \int_{\tau}^{\tau+h} F(t, x(t)) d t .
$$

Hence

$$
\begin{aligned}
& \operatorname{dist}((x(\tau+h)-x(\tau)) / h, F(\tau, x(\tau))) \\
& \leqq \operatorname{Dist}\left(\frac{1}{h} \int_{\tau}^{\tau+h} F(t, x(t)) d t, F(\tau, x(\tau))\right) \\
& \leqq \operatorname{Dist}\left(\frac{1}{h} \int_{E_{h}(\tau)} F(t, x(t)) d t, \frac{1}{h} \int_{E_{h}(\tau)} F(\tau, x(\tau)) d t\right) \\
& +\operatorname{Dist}\left(\frac{1}{h} \int_{H_{h}(\tau)} F(t, x(t)) d t, \frac{1}{h} \int_{H_{h}(\tau)} F(\tau, x(\tau)) d t\right) \\
& \leqq \frac{1}{|h|} \int_{E_{h}(\tau)} \operatorname{Dist}(F(t, x(t)), F(\tau, x(\tau))) d t \\
& +\frac{1}{|h|} \int_{H_{h}(\tau)} k(t) d t+|F(\tau, x(\tau))| \frac{\operatorname{meas}\left(H_{h}(\tau)\right)}{|h|} .
\end{aligned}
$$

Since the last term tends to zero with $h$, the following relation holds

$$
D^{*} x(\tau) \subset F(\tau, x(\tau)) \text {. }
$$


A positive number $\varepsilon$ is arbitrary, and hence for almost every $\tau$ in $I$ the relation

$$
D^{*} x(\tau) \subset F(\tau, x(\tau))
$$

holds for every trajectory $x(t)$ of $F(t, x)$.

\section{REFERENCES}

[1] Kikuchi. N.. On some fundamental theorems of contingent equations in connection with the control problems. Publ. RIMS, Kyoto Univ. Ser. A, 3 (1967), 177-201.

[2] Opial, Z., Sur l'équation différentielle ordinaire du premier ordre dont le second membre satisfait aux conditions de Carathéodory, Ann. Polon. Math. 8 (1960). 23-28.

[3] Scorza-Dragoni, G., Un teorem sulle funzioni continue rispetto ad une e misurabili rispetto ad unaltra variable. Rend. Sem. Mat. Univ. Padova. 17 (1948). 102-106. 
